\section{Kidney \\ Blood Pressure Research}

\title{
Serum Fas Ligand, Serum Myostatin and Urine TGF- $\beta 1$ Are Elevated in Autosomal Dominant Polycystic Kidney Disease Patients with Impaired and Preserved Renal Function
}

\author{
Vassilios Raptis ${ }^{\mathrm{a}, \mathrm{b}} \quad$ Constantinos Bakogiannis ${ }^{c} \quad$ Charalampos Loutradis $^{\mathrm{a}}$ \\ Afroditi K. Boutou $^{d} \quad$ Athanasios Sioulis $^{\mathrm{b}}$ Elias Balaskas ${ }^{\mathrm{b}}$ Pantelis Zebekakis ${ }^{\mathrm{b}}$ \\ Pantelis Sarafidis ${ }^{a}$

\begin{abstract}
aDepartment of Nephrology, Hippokration Hospital, Aristotle University of Thessaloniki, Thessaloniki, bSection of Nephrology and Hypertension, 1st Department of Medicine, AHEPA Hospital, Aristotle University of Thessaloniki, Thessaloniki, c $3^{\text {rd }}$ Department of Cardiology Hippokration Hospital, Aristotle University of Thessaloniki, Thessaloniki, dDepartment of Respiratory Medicine, Papanikolaou Hospital, Thessaloniki, Greece
\end{abstract}

\section{Key Words}

Fibrosis • Apoptosis • Endothelial dysfunction • Chronic kidney disease

\begin{abstract}
Background/Aims: The pathophysiology of renal disease progression in autosomal dominant polycystic disease (ADPKD) is largely unknown. Recent evidence suggests microvascular dysfunction leading to renal ischemia, as an additional pathway for renal function decline. This study examined the levels of serum Fas ligand (FasL), serum myostatin and urine transforming growth factor-beta 1 (TGF- $\beta 1$ ) and their association with markers of endothelial dysfunction, in ADPKD patients with preserved or impaired renal function. Methods: Seventy-eight participants were enrolled in the study, divided in three groups: Group A consisted of 26 ADPKD patients with impaired renal function (eGFR $45-70 \mathrm{ml} / \mathrm{min} / 1.73 \mathrm{~m}^{2}$ ), Group B of 26 ADPKD patients with preserved renal function (eGFR $>70 \mathrm{ml} / \mathrm{min} / 1.73 \mathrm{~m}^{2}$ ), and Group $C$ of 26 age- and sex- matched controls with no history of renal disease. Serum FasL, myostatin and urine levels of TGF- $\beta 1$ were measured as biomarkers of vascular dysfunction, apoptosis and fibrosis with ELISA techniques. Results: Group A patients had significantly higher levels of FasL $(13.12 \pm 1.69 \mathrm{ng} / \mathrm{mL})$, myostatin $(4.62 \pm 0.59 \mathrm{ng} / \mathrm{mL})$ and urine logTGF- $\beta 1(3.56 \pm 0.49$ $\mathrm{ng} / 24 \mathrm{~h})$ compared to Group B $(9.6 \pm 1.28 \mathrm{ng} / \mathrm{mL}, 3.06 \pm 0.35$, and $2.09 \pm 0.37$, respectively, $\mathrm{p}<0.001$ for all comparisons) or controls $(6.59 \pm 1.17 \mathrm{ng} / \mathrm{mL}, 2.18 \pm 0.45 \mathrm{ng} / \mathrm{ml}$, and $1.58 \pm 0.21$,
\end{abstract}




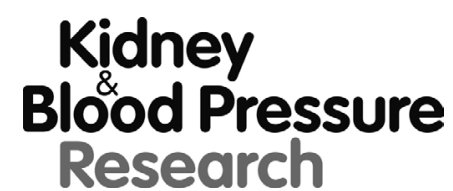

Kidney Blood Press Res 2018;43:744-754

\begin{tabular}{|l|l|}
\hline DOI: $10.1159 / 000489911$ & (c) 2018 The Author(s). Published by S. Karger AG, Basel \\
\hline
\end{tabular}

Published online: 22 May, 2018

www.karger.com/kb

Raptis et al.: Apoptosis, Fibrosis and Endothelial Dysfunction in ADPKD

respectively, $p<0.001$ for all comparisons). Patients in Group B had also higher levels of all markers compared to controls $(p<0.001)$, despite having similar renal function. In ADKPD patients negative associations of eGFR with FasL $(r=-0.799, p<0.001)$, myostatin $(r=-0.856$, $p<0.001)$ and TGF- $\beta 1(r=-0.476, p<0.001)$ but positive correlations of these markers with asymmetric dimethylarginine (ADMA) $(r=0.825 ; r=0.749$; and $r=0.599$, respectively $p<0.001)$ were noted. Multivariate analysis demonstrated that FasL was independently associated with high urine TGF- $\beta 1$ (OR 3.774, 95\%CI 1.180-12.072, $\mathrm{p}=0.025$ ). Conclusions: ADPKD patients with moderately preserved renal function have higher levels of FasL, myostatin and urine TGF- $\beta 1$ than controls. These results indicate that an interplay between endothelial dysfunction and renal ischemia with mechanisms linked to apoptosis and fibrosis may be present even in early stages of ADPKD.

(C) 2018 The Author(s)

Published by S. Karger AG, Basel

\section{Introduction}

Over the last decades, renal cyst formation and cyst growth have been considered as the key mechanism leading to renal failure in autosomal dominant polycystic kidney disease (ADPKD) [1,2]. Renal cyst formation in ADPKD is caused by dysfunctional polycystin proteins, related to mutations of PKD1 and PKD2 genes, which encode polycystin 1 and polycystin 2 respectively [3]. Apart from the recent exception with the use of tolvaptan, a vasopressin2-receptor antagonist, which was associated with retardation of renal function decline [4], previous therapeutic efforts with medications, such as the m-TOR inhibitor everolimus, or with surgical drainage of cysts, failed to or delay the progression of renal disease in ADPKD patients [5]. As ADPKD remains the fourth cause of end-stage renal disease [6] understanding of the pathophysiology of renal function decline could further drive our efforts to design optimal therapeutic strategies for ADPKD patients.

In previous years different hypotheses on the cystogenesis processes in ADPKD have been described [7]; existing data support that a complete loss of the normal allele is required (two-hit hypothesis), whereas other support that cysts can develop only with the level of function protein falling below a specific level (threshold hypothesis) [7]. In PKD1 inducible knockout (IKO) rats, PKD1 gene inactivation resulted in the formation of focal cysts only after induction of ischemia-reperfusion [8]. In adult mice with inactivated PKD1 gene showed, nephrotoxic injury led to increased proliferation of tubular epithelial cells and accelerated the formation of cysts [9]. Such observations suggest that the presence of abnormal polycystins, or reduced expression of the normal proteins, lead to renal cyst formation, but other mechanisms, such as kidney injury from ischemia-reperfusion or other nephrotoxic, paracrine or inflammatory factors that promote tubular damage initiation, activation of the apoptotic process, and increased cell proliferation, can accelerate cystogenesis [10]. Furthermore, polycystins are present not only in renal tubular cells, but also in the primary cilia of endothelial cells of renal microvasculature, where they function as flow sensors [11]. Impaired expression of normal polycystins in cultured endothelial cells was associated with an abnormal intracellular response to shear stress; i.e. inability to transmit extracellular shear stress into intracellular calcium signaling and NO synthesis [12]. It was therefore hypothesized that the "dysfunctional" polycystins, which per se exist in ADPKD, induce a pathological mechanosensory response to the vascular blood flow changes causing oxidative stress and ADMA increase, instead of NO production. Reduced bioavailability of NO may lead to vascular remodeling, and renal ischemia, which may act as an additional mechanism promoting the apoptotic process, the increased proliferation and the increased production of basic matrix and production of the matrix, resulting in fibrosis, further creating a vicious circle of ischemia, hypoxia, fibrosis and progressive worsening of renal function [5].

We have recently demonstrated that ADPKD patients with relatively preserved renal function have significantly increased circulating asymmetric dimethylarginine (ADMA) levels, a marker of endothelial dysfunction [13]. ADMA levels in this group of patients were 


\section{Kidney Blood Pressure Research}

Raptis et al.: Apoptosis, Fibrosis and Endothelial Dysfunction in ADPKD

positively associated with oxidative stress biomarkers, as well as with biomarkers of hypoxia and angiogenesis such as HIF-1a and angiopoietin-2 [13]. Apoptosis is a field extensively studied in various diseases, thus there are a number of biomarkers linked to apoptosis. Fas ligand (FasL) is a transmembrane protein, member of the tumor necrosis factor, which induces apoptosis via various molecular mechanisms after its binding with its receptor Fas $[14,15]$. Though FasL participates in various processes, its circulating levels, as well as soluble Fas levels, have been extensively used as markers indicative of apoptotic and inflammatory activity in various diseases, including kidney disease [16-20]. Myostatin is a transforming growth factor-beta (TGF-beta) superfamily member, closely associated with skeletal muscle differentiation. It has been demonstrated that myostatin could elicit apoptotic mechanisms via complex signaling pathways such inducing G1 phase cell cycle arrest, while increased myostatin levels are associated with endothelial dysfunction and renal decline in diabetic nephropathy $[17,21]$. In addition, it has been recently demonstrated that myostatin circulating levels were elevated from early stages of CKD and inversely related to eGFR [22]. Moreover, fibrosis elicited both via cysts, inflammatory cytokines, or mechanistic processes, is also a key step in renal function decline in ADPKD. Urine TGF- $\beta 1$ levels have been used as a marker reflecting fibrotic changes [23-25].

Based on the above, the aim of this study was to determine the levels of apoptosis-related biomarkers, namely FasL and myostatin, not adequately studied in ADPKD, in relation to a marker of renal fibrosis, urine levels of TGF- $\beta 1$, in APDKD patients with moderately preserved renal function, and compare them with ADPKD patients with impaired renal function and control subjects. Furthermore, we want to examine possible associations between these biomarkers and indices of endothelial dysfunction ADPKD patients.

\section{Materials and Methods}

\section{Study design}

We prospectively evaluated ADPKD patients attending the Nephrology Outpatient Clinics of AHEPA University Hospital, Thessaloniki Greece, and General Hospital of Pieria, Katerini, Greece during the years 2010 and 2011, for possible inclusion in the study, as previously described [13]. Inclusion criteria were: a) documented diagnosis of ADPKD, according to standard ultrasonographic criteria [26] and positive family history of the disease b) age 18-65 years; c) provision of informed written consent. Patients were excluded from the study if they had: a) CKD stage 3 or higher, (i.e. estimated glomerular filtration rate (eGFR) $<45 \mathrm{ml} /$ min/1.73m², according to the Kidney Disease Improving Global Outcomes (KDIGO) 2012 Guideline for the Evaluation and Management of Chronic Kidney Disease [27]; b) acute or chronic inflammatory disease; c) current use of cyclooxygenase inhibitors, antioxidants or vitamin E supplements, d) history of complicated renal cysts in the 3 previous months prior to study enrollment; e) acute myocardial infarction, unstable angina or ischemic stroke during the past 3 months; f) evidence of peripheral occlusive arterial disease; g) stage III-IV heart failure, according to the New York Heart Association (NYHA) criteria [28].

A total of 52 patients with ADPKD fulfilled the aforementioned inclusion/exclusion criteria and were included in the study. Based on eGFR levels, two groups of ADPKD patients were formed: first, Group A, which included 26 patients with modestly impaired renal function i.e. eGFR ranging from 45 to $70 \mathrm{ml} /$ $\mathrm{min} / 1.73 \mathrm{~m}^{2}$ and Group B, which included 26 age- and sex-matched patients with relatively preserved renal function i.e., eGFR $>70 \mathrm{ml} / \mathrm{min} / 1.73 \mathrm{~m}^{2}$. A third group, Group $\mathrm{C}$, including 26 age- and sex-matched volunteers who were apparently healthy or had mild hypertension and normal serum creatinine levels, formed the control group of the study.

All protocol procedures were conducted in accordance with the Declaration of Helsinki (2000 amendment). The study protocol was approved by the Ethics Committee of School of Medicine, Aristotle University of Thessaloniki. Participants provided informed written consent prior to study initiation. Screening evaluation included detailed medical history, physical examination and routine laboratory tests. At the main study visit, participants' demographics, medical history, and medication were recorded. Height, weight and blood pressure (BP) measurements were also recorded in a standardized manner, as per clinical 


\section{Kidney Blood Pressure Research}

Raptis et al.: Apoptosis, Fibrosis and Endothelial Dysfunction in ADPKD

routine. All study subjects were instructed to bring in the outpatient clinic a 24-hour urinary collection to determine urinary albumin excretion. Morning blood samples following a 12-hour overnight fasting were drawn to determine routine hematological and biochemical labs as well as circulatory markers under study.

\section{Laboratory analyses}

Routine hematological and biochemical parameters were measured with standard laboratory methods. eGFR was estimated from serum creatinine levels with the Modification of Diet in Renal Disease (MDRD) equation, [29]. For the determination of biomarkers under study blood samples were collected in standard EDTA tubes, were immediately centrifuged, and the supernatants were stored at $-70^{\circ} \mathrm{C}$. 24 -hour urine samples for TGF- $\beta 1$ determination were similarly stored. Circulating levels of FasL were determined with the R\&D systems human Fas Ligand/TNFSF6 Quantikine ELISA (intra-assay CV: 4.1-5.4\%, inter-assay CV: 6.4-8.8\%), myostatin with the R\&D systems GDF-8/Myostatin Quantikine ELISA (intra-assay CV: 1.8-5.4\%, inter-assay CV: $3.1-6.0 \%$ ), and urine TGF- $\beta 1$ with the R\&D systems human TGF- $\beta 1$ Quantikine ELISA (intraassay CV: $2.4-3.4 \%$, inter-assay CV: $5.7-84 \%)$.

\section{Statistical analysis}

Categorical variables are expressed as absolute frequencies and percentages and continuous variables as mean \pm standard deviation (mean \pm SD). We used the Chi Square $(\chi 2)$ test or Fisher's exact test for betweengroups comparisons for categorical variables. For continuous variables we used one-way analysis of variance (ANOVA) and Student's $t$ tests for independent variables to examine differences between various pairs of the three study groups. Variables were assessed for distribution analysis by Shapiro-Wilk testing. TGF- $\beta 1$ in 24-hour urine collections was log-transformed for parameter testing. We performed simple linear regression analysis and calculated Pearson's $r$ correlation coefficients, to explore the possible associations between biomarkers under study and eGFR, as well as levels of ADMA studied in the same population and published in a previous report [13]. Furthermore, univariate and two separate models of multivariate regression analysis (one with ADMA and a second with FasL), were performed, in order to evaluate possible associations between clinical and demographic factors and these specific biomarkers with higher levels of urine TGF- $\beta 1$ levels. Statistical analysis was performed using the Statistical Package for Social Sciences (SPSS) software version 17 (SPSS Inc, Chicago, Ill, USA) for Windows XP. A p value of $<0.05$ (two-tailed) was considered statistically significant.

\section{Results}

\section{Baseline characteristics}

Baseline clinical and laboratory characteristics, as well as medication data for the study population are shown in Table 1. There were no significant differences between groups with respect to age, sex, as per study design. Group A patients had significantly higher levels of serum urea and creatinine, and significantly lower eGFR levels when compared to Group B patients and controls. Furthermore, Group A patients were treated with significantly more antihypertensive agents $(2.7 \pm 1.7)$, when compared to Group B patients $(1.4 \pm 1.1)$ or controls $(0.6 \pm 0.2) \quad(p<0.001$ for all comparisons). On the other hand, patients with ADPKD and preserved renal function (Group B) did not differ from controls in terms of serum urea and creatinine, eGFR, urinary albumin excretion, BP and uric acid. Finally, Group A patients had significantly higher ADMA levels compared to the other two groups $(p<0.001)$ and Group B patients, i.e. those with preserved renal function, compared to controls $(p<0.001)$ [13].

\section{Serum levels of FasL and myostatin}

Table 2 and Fig. 1 present serum levels of FasL and myostatin, and urine levels of TGF- $\beta 1$. Group A, i.e. ADPKD patients with impaired renal function had significantly higher levels of FasL $(13.12 \pm 1.69 \mathrm{ng} / \mathrm{mL})$ compared to Group B patients $(9.6 \pm 1.28 \mathrm{ng} / \mathrm{mL}, \mathrm{p}<0.001)$ or controls $(6.59 \pm 1.17 \mathrm{ng} / \mathrm{mL}, \mathrm{p}<0.001)$. Myostatin levels were also higher in Group A patients $(4.62 \pm 0.59 \mathrm{ng} / \mathrm{mL})$ when compared to Group B patients $(3.06 \pm 0.35 \mathrm{ng} / \mathrm{mL}, \mathrm{p}<0.001)$ or 


\section{Kidney Blood Pressure Research}

Table 1. Baseline demographic, laboratory and pharmaceutical treatment characteristics of study groups. $\mathrm{ADPKD}=$ autosomal dominant polycystic kidney disease; eGFR=estimated glomerular filtration rate; hsCRP: high sensitivity $\mathrm{C}$ reactive protein; $\mathrm{SBP}=$ systolic blood pressure; $\mathrm{DBP}=$ diastolic blood pressure; $\mathrm{ACEI}=$ angiotensin converting enzyme inhibitors; $\mathrm{ARBs}=$ angiotensin receptor blockers; $\mathrm{CCB}=$ calcium channel blockers. Data are presented as mean \pm SD or absolute and relevant frequencies

\begin{tabular}{|c|c|c|c|c|}
\hline Parameter & ADPKD with impaired renal function & ADPKD with preserved renal function & Controls & P-values \\
\hline $\mathrm{N}$ & 26 & 26 & 26 & \\
\hline Age (years) & $44.0 \pm 12.1$ & $43.0 \pm 11.2$ & $43.5 \pm 11.2$ & 0.962 \\
\hline \multicolumn{5}{|l|}{ Sex } \\
\hline Male, n (\%) & $13(50.0 \%)$ & $13(50.0 \%)$ & $13(50.0 \%)$ & \multirow{2}{*}{1.000} \\
\hline Female, n (\%) & $13(50.0 \%)$ & $13(50.0 \%)$ & $13(50.0 \%)$ & \\
\hline Weight (kg) & $73.0 \pm 13.7$ & $80.8 \pm 17.1$ & $69.5 \pm 12.0$ & 0.019 \\
\hline Hemoglobin $(\mathrm{g} / \mathrm{dl})$ & $13.3 \pm 1.0$ & $13.2 \pm 0.7$ & $12.9 \pm 1.0$ & 0.388 \\
\hline Serum urea $(\mathrm{mg} / \mathrm{dl})$ & $68.9 \pm 21.9$ & $42.9 \pm 18.2$ & $32.7 \pm 8.7$ & $<0.001$ \\
\hline Serum creatinine $(\mathrm{mg} / \mathrm{dl})$ & $1.8 \pm 0.5$ & $1.1 \pm 0.4$ & $1.0 \pm 0.3$ & $<0.001$ \\
\hline eGFR $\left(\mathrm{ml} / \mathrm{min} / 1.73 \mathrm{~m}^{2}\right)$ & $51.7 \pm 5.0$ & $89.5 \pm 13.1$ & $90.8 \pm 10.1$ & $<0.001$ \\
\hline Uric acid (mg/dl) & $6.7 \pm 0.8$ & $6.0 \pm 0.8$ & $5.8 \pm 0.5$ & $<0.001$ \\
\hline ADMA $(\mu \mathrm{mol} / \mathrm{l})$ & $1.68 \pm 0.7$ & $1.26 \pm 0.7 *$ & $0.51 \pm 0.2$ & $<0.001$ \\
\hline $\mathrm{hsCRP}(\mathrm{mg} / \mathrm{dl})$ & $1.4 \pm 0.2$ & $0.7 \pm 0.2$ & $0.2 \pm 0.1$ & $<0.001$ \\
\hline Urinary albumin excretion (mg/day) & $340.5 \pm 56.6$ & $145.0 \pm 57.2$ & $15.4 \pm 11.0$ & $<0.001$ \\
\hline Presence of albuminuria, $n(\%)$ & $19(73.1 \%)$ & $8(30.8 \%)$ & $2(7.7 \%)$ & 0.087 \\
\hline Presence of hematuria, n (\%) & $17(65.4 \%)$ & $18(69.2 \%)$ & $0(0.0 \%)$ & $<0.001$ \\
\hline Age onset of hematuria (years) & $31.2 \pm 9.9$ & $35.0 \pm 10.2$ & - & 0.270 \\
\hline History of urine track infection, $n(\%)$ & $12(46.2 \%)$ & $13(50.0 \%)$ & $9(34.6 \%)$ & 0.266 \\
\hline Current smokers, n (\%) & $15(57.7 \%)$ & $13(50.0 \%)$ & $13(50.0 \%)$ & 0.814 \\
\hline History of hypertension, n (\%) & $17(65.4 \%)$ & $20(76.9 \%)$ & $13(50.0 \%)$ & 0.154 \\
\hline Age onset of hypertension, $\mathrm{n}(\%)$ & $32.9 \pm 11.3$ & $35.4 \pm 9.0$ & $41.9 \pm 13.8$ & 0.098 \\
\hline SBP $(\mathrm{mmHg})$ & $135.2 \pm 7.3$ & $132.0 \pm 7.1$ & $129.8 \pm 7.8$ & 0.036 \\
\hline DBP (mmHg) & $80.4 \pm 10.2$ & $83.3 \pm 5.4$ & $79.0 \pm 7.1$ & 0.145 \\
\hline Number of antihypertensive agents & $2.7 \pm 1.7$ & $1.4 \pm 1.1$ & $0.6 \pm 0.2$ & $<0.001$ \\
\hline ACEIs, n (\%) & $15(57.7 \%)$ & $10(38.4 \%)$ & $5(19.2 \%)$ & 0.157 \\
\hline ARBs, n (\%) & $13(50.0 \%)$ & $7(26.9 \%)$ & $4(15.4 \%)$ & 0.370 \\
\hline CCBs, n (\%) & $2(7.7 \%)$ & $0(0.0 \%)$ & $1(3.8 \%)$ & 0.474 \\
\hline$\beta$-blockers, n (\%) & $15(57.7 \%)$ & $11(42.3 \%)$ & $5(19.2 \%)$ & 0.091 \\
\hline Statins, n (\%) & $11(42.3 \%)$ & $6(23.1 \%)$ & $3(11.5 \%)$ & 0.344 \\
\hline
\end{tabular}

Table 2. Levels of FasL, myostatin and urine TGF- $\beta 1$ in the three study groups. * P-values for the comparison between Group A (ADPKD patients with impaired renal function) vs. Group B (ADPKD patients with preserved renal function). ${ }^{* *}$ P-values for the comparison between Group A (ADPKD patients with impaired renal function) vs. Group C (controls). † P-values for the comparison between Group B (ADPKD patients with preserved renal function) vs. Group C (controls). ADPKD= autosomal dominant polycystic kidney disease; FasL: Fas ligand; TGF- $\beta 1$ : transforming growth factor- beta 1

\begin{tabular}{|c|c|c|c|c|c|c|}
\hline Parameter & Group A & Group B & P- & Group C & $\mathrm{P}-$ & $\mathrm{P}-$ \\
\hline $\mathrm{N}$ & 26 & 26 & & 26 & & \\
\hline serum FasL (ng/ml) & $13.12 \pm 1.69$ & $9.6 \pm 1.28$ & $<0.001$ & $6.59 \pm 1.17$ & $<0.001$ & $<0.001$ \\
\hline serum Myostatin & $4.62 \pm 0.59$ & $3.06 \pm 0.35$ & $<0.001$ & $2.18 \pm 0.45$ & $<0.001$ & $<0.001$ \\
\hline urine logTGF- $\beta 1$ & $3.56 \pm 0.49$ & $2.09 \pm 0.37$ & $<0.001$ & $1.58 \pm 0.21$ & $<0.001$ & $<0.001$ \\
\hline
\end{tabular}

controls $(2.18 \pm 0.45 \mathrm{ng} / \mathrm{mL}, \mathrm{p}<0.001)$. Of note, Group B patients, i.e. those with relatively preserved renal function had also significantly higher levels of apoptotic biomarkers when compared to controls (FasL: $9.6 \pm 1.28$ vs. $6.59 \pm 1.17 \mathrm{ng} / \mathrm{mL}, \mathrm{p}<0.001$; myostatin: $3.06 \pm 0.35$ vs. $2.18 \pm 0.45 \mathrm{ng} / \mathrm{mL}, \mathrm{p}<0.001)$.

\section{Levels of urine TGF- $\beta 1$}

Patients with impaired renal function (Group A) had significantly higher levels of $\log$ TGF- $\beta 1$ in 24-hour urine collections $(3.56 \pm 0.49 \mathrm{ng} / 24 \mathrm{~h})$, when compared to patients from Group B (2.09 $\pm 0.37 \mathrm{ng} / 24 \mathrm{~h}, \mathrm{p}<0.001)$, or controls $(1.58 \pm 0.21 \mathrm{ng} / 24 \mathrm{~h}, \mathrm{p}<0.001)$. Similar to above, urine TGF- $\beta 1$ was also significantly higher in patients with moderately preserved renal function compared to controls $(\mathrm{p}<0.001)$, despite similar eGFR of these two groups (Table 2 and Fig. 1).

\section{Correlation and regression analyses}

In order to further examine the role of decreased renal function with the evolution of apoptosis and fibrosis, we examined in correlation analyses, associations between FasL, myostatin and TGF- $\beta 1$ with eGFR in ADPKD patients (Fig. 2). We observed a negative correlation of eGFR with FasL $(r=-0.799, \mathrm{p}<0.001)$, myostatin $(\mathrm{r}=-0.856, \mathrm{p}<0.001)$ and TGF- $\beta 1(r=-0.476, p<0.001)$. To study the interaction endothelial dysfunction with apoptotic 


\section{Kidney Blood Pressure Research}

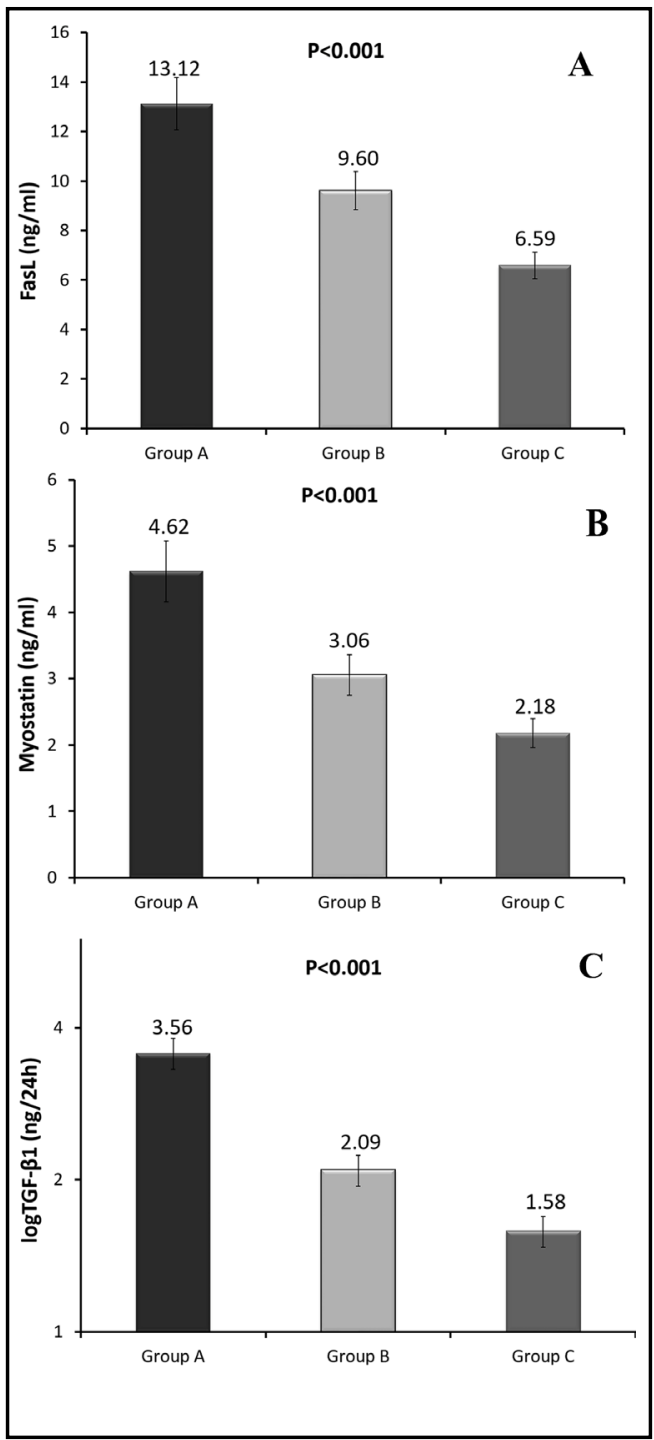

Fig. 1. Levels of: a) serum FasL, b) serum myostatin and c) urine TGF- $\beta 1$ in patients with ADPKD patients with impaired renal function (Group A), ADPKD patients with preserved renal function (Group B) and controls (Group C).

and fibrotic processes, we examined the correlations of ADMA, an established marker of endothelial dysfunction with FasL, myostatin and TGF- $\beta 1$, observing significant positive correlations in all three cases ( $\mathrm{r}=0.825, \mathrm{p}<0.001 ; \mathrm{r}=0.749, \mathrm{p}<0.001$; and $\mathrm{r}=0.599, \mathrm{p}<0.001$, respectively) (Fig. 3 ).

We also performed univariate and multivariate analyses using increased urine TGF- $\beta 1$ as dependent variable (Table 3). In univariate analysis decrease in eGFR, and increase in uric acid, ADMA, and FasL were associated with increased TGF- $\beta 1$ levels. Due to the significant interaction between ADMA and FasL, we constructed two different multivariate models with these variables. In the first eGFR continued to be inversely associated with TGF- $\beta 1$ [Odds Ratio (OR) 0.862, 95\% Confidence Intervals (CI) 0.784-0.948)], while ADMA had a marginally significant association (OR 5.139, 95\%CI 0.887-29.768). In the second model
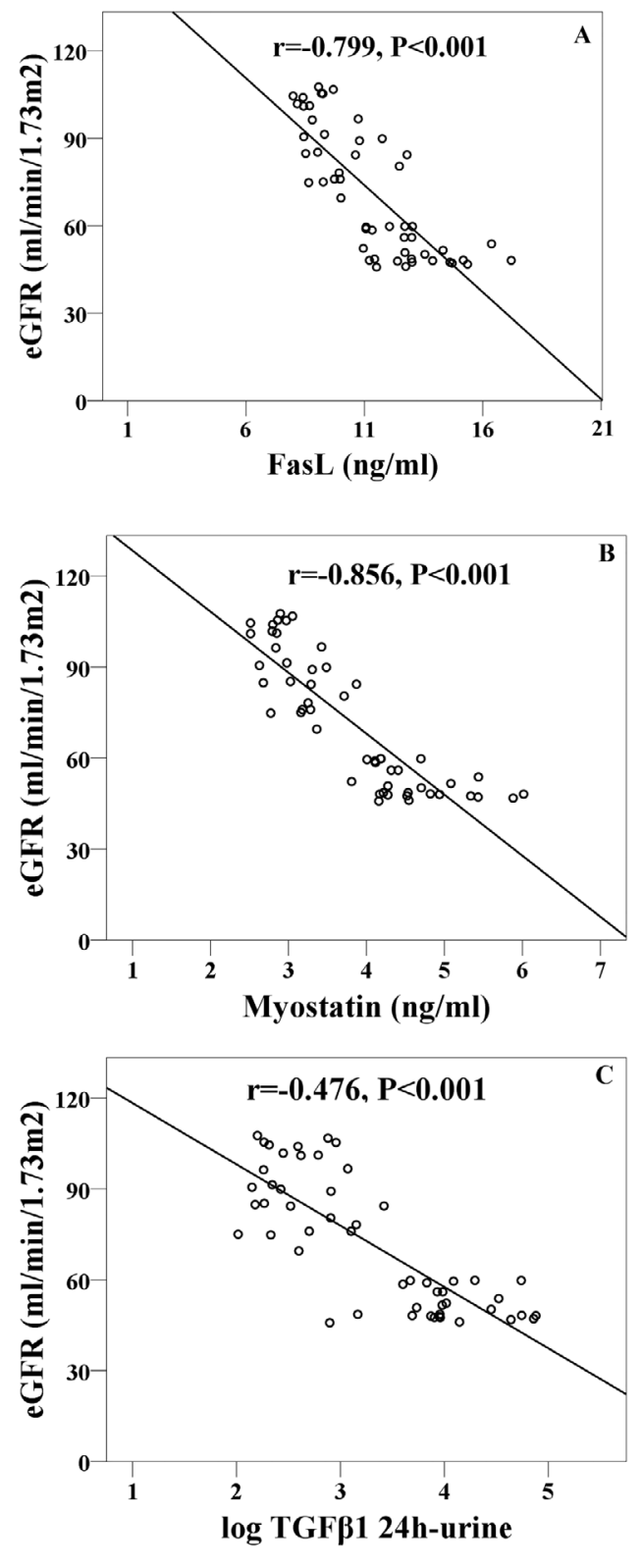

Fig. 2. Correlation analysis between eGFR and levels of a) serum FasL $(r=-0.799, p<0.001)$ b) serum myostatin $(r=-0.856, p<0.001)$, and $c)$ urine TGF- $\beta 1$ $(\mathrm{r}=-0.476, \mathrm{p}<0.001)$ in ADPKD patients $(\mathrm{n}=52)$. 


\section{Kidney \\ Blood Pressure Research}

Kidney Blood Press Res 2018;43:744-754

\begin{tabular}{l|l}
\hline DOI: $10.1159 / 000489911$ & (C) 2018 The Author(s). Published by S. Karger AG, Basel
\end{tabular}
FasL was the only parameter continuing to be significantly associated with high urine TGF- $\beta 1$ levels (OR 3.774, 95\%CI 1.18012.072).

\section{Discussion}

The aim of this study was to examine the levels of FasL, myostatin and urine TGF- $\beta 1$, biomarkers related to apoptosis and fibrosis, in patients with ADPKD with different stages of renal function, and how these correlate with markers of endothelial function. We examined apoptosis related biomarkers, not studied in ADPKD, i.e. FasL and myostatin, as well as, urine levels of TGF- $\beta 1$ in carefully matched ADPKD patients with impaired and preserved renal function, and controls. The main finding of our study was a stepwise increase of all these biomarkers from controls to ADPKD patients with preserved and those with impaired renal function. This significant increase in ADPKD patients with preserved renal function was present despite the fact that eGFR levels were similar between this group and controls. In addition, all parameters under study exhibited strong inverse correlations with eGFR and positive correlations with ADMA, an established marker of endothelial dysfunction. In multivariate analysis FasL was independently associated with TGF- $\beta 1$, indicating the strong connection between apoptotic and fibrotic phenomena.

The mechanism of renal disease progression in APDKD patients remains a topic of great interest. The initial hypothesis that growth of cysts due to their mechanical effect on renal parenchyma is the only factor responsible is not valid [1, $2]$, as this can only partially explain the worsening of renal function in APDKD patients. Therapeutic interventions, using surgical or pharmacological techniques to reduce cysts' growth also failed to adequately reverse ADPKD progression [5], with the exception of the recent Replicating Evidence of Preserved Renal Function: an Investigation of Tolvaptan Safety and Efficacy in ADPKD (REPRISE) trial, where tolvaptan was associated with slower eGFR decline compared with placebo $(-2.34$ versus $-3.61 \mathrm{~mL} / \mathrm{min} / 1.73 \mathrm{~m} 2, \mathrm{P}<0.001$ ) [4].

Reduced renal blood flow, via or and endothelial function and vasoconstriction, could be attributed to various factors in ADPKD, such as increased activation of renin-angiotensinaldosterone system, increased sympathetic activation, decreased nitric oxide bioavailability, and others [30]. We have previously demonstrated that ADMA, an endogenous inhibitor of
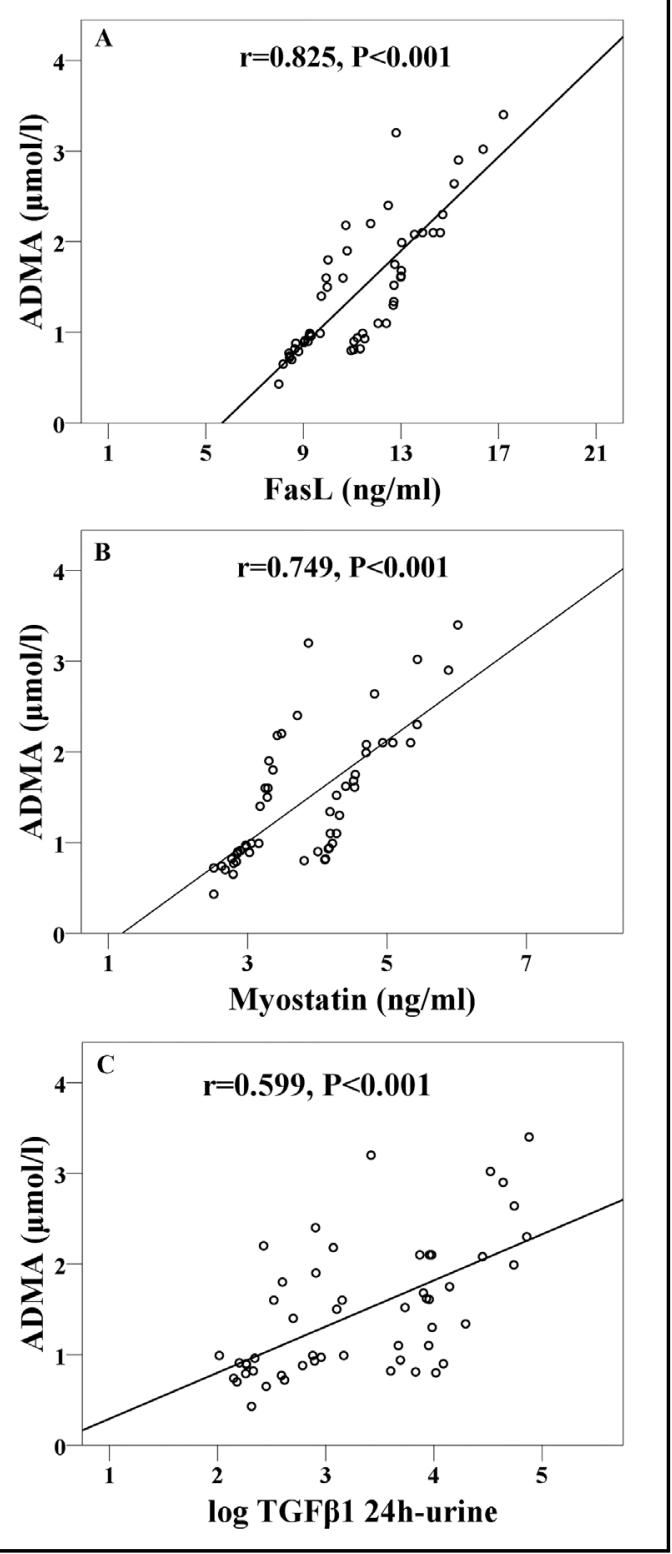

Fig. 3. Correlation analysis between ADMA and levels of a) serum FasL ( $r=0.825, \mathrm{p}<0.001)$ b) serum myostatin ( $r=0.749, \mathrm{p}<0.001)$, and $\mathrm{c}$ ) urine TGF- $\beta 1$ $(r=0.599, p<0.001)$ in ADPKD patients $(n=52)$. 


\section{Kidney Blood Pressure Research}

Table 3. Univariate and multivariate regression analyses on the association of traditional clinical and laboratory factors, as well as ADMA or FasL with urine TGF- $\beta 1$ levels in ADPKD patients. ADMA: asymmetric dimethylarginine, eGFR=estimated glomerular filtration rate; FasL: Fas ligand, SBP=systolic blood pressure; $\mathrm{DBP}=$ diastolic blood pressure; hsCRP: high sensitivity $\mathrm{C}$ reactive protein;. Data are presented as Odds Ratio $(95 \% \mathrm{CI})$

\begin{tabular}{|c|c|c|c|c|c|c|}
\hline \multirow[b]{2}{*}{ Parameter } & \multicolumn{2}{|c|}{ Univariate analysis } & \multirow{2}{*}{$\begin{array}{c}\text { Multivariate analysis with } \\
\text { Adjusted Odds Ratio }\end{array}$} & \multicolumn{3}{|c|}{ Multivariate analysis with } \\
\hline & Unadjusted Odds & $\mathrm{P}$ & & $\mathrm{P}$ & Adjusted Odds Ratio & $\mathrm{P}$ \\
\hline Age (per year increase) & $1.005(0.956-1.056)$ & 0.846 & & & & \\
\hline Male Gender & $0.711(0.226-2.241)$ & 0.561 & & & & \\
\hline Hypertension & $1.083(0.305-3.850)$ & 0.902 & & & & \\
\hline Dyslipidemia & $1.467(0.457-4.706)$ & 0.520 & & & & \\
\hline SBP (per mmHg increase) & $1.080(0.991-1.176)$ & 0.079 & $1.115(0.929-1.338)$ & 0.242 & $1.068(0.911-1.252)$ & 0.416 \\
\hline DBP (per mmHg increase) & $0.984(0.918-1.055)$ & 0.646 & & & & \\
\hline eGFR (per $\mathrm{ml} / \mathrm{min} / 1.73 \mathrm{~m}^{2}$ & $0.865(0.791-0.947)$ & 0.002 & $0.931(0.848-1.021)$ & 0.130 & $0.862(0.784-0.948)$ & 0.002 \\
\hline Uric Acid (per mg/dl increase) & $2.158(1.037-4.491)$ & 0.040 & $1.211(0.388-3.776)$ & 0.742 & $1.160(0.395-3.406)$ & 0.788 \\
\hline hsCRP (per mg/dl increase) & $1.694(0.903-3.180)$ & 0.101 & $0.894(0.372-2.150)$ & 0.803 & $1.109(0.535-2.298)$ & 0.781 \\
\hline ADMA (per $\mu \mathrm{mol} / \mathrm{l}$ increase) & $4.215(1.603-11.080)$ & 0.004 & $5.139(0.887-29.768)$ & 0.068 & - & \\
\hline FasL (per ng/ml increase) & $4.025(1.812-8.941)$ & 0.001 & - & & $3.774(1.180-12.072)$ & 0.025 \\
\hline
\end{tabular}

nitric oxide, was significantly increased in ADPKD, even from the early stages of the disease, and this increase was linked to increased levels of oxidative stress [13]. Further to this observation, we have also showed that ADPKD patients with preserved and moderately impaired renal function, exhibited higher levels of emerging biomarkers indicative of endothelial dysfunction, angiogenesis and hypoxia i.e. endocan, hypoxia inducible factor1a (HIF-1a) and angiopoietin-2 (Ang-2) [31]. In addition, we have showed that levels of endocan, HIF-1a and Ang-2 were positively correlated with ADMA in patients with ADPKD. As a consequence, we have hypothesized, that endothelial dysfunction, via disturbances in local renal microcirculation leading to ischemia, hypoxia and angiogenesis, could act as regulator for renal injury progression in patients at early stages of the disease [5, 31].

Morphological and structural changes observed in advanced stages of ADPKD include cell proliferation and interstitial fibrosis, closely linked to activation of apoptotic processes in renal microenvironment, as has been previously extensively described [30]. We studied FasL and myostatin in our population. Although there are no data in APDKD, Yilmaz et al. [17], demonstrated that in patients with diabetic nephropathy and chronic kidney disease Stage 1 soluble Fas (sFas), the counterpart of FasL, and myostatin were significantly related to both ADMA levels, as well as decreases in flow mediated dilatation (FMD), as markers of endothelial dysfunction. In a recent cross-sectional study in 781 participants of a health exam, myostatin was elevated from early CKD stages an inversely related with eGFR [22]. Recent data indicated that myostatin is expressed and localized in human peri-renal aortic tissue specimens, participating in atherosclerotic plaques development via activation of JNK pathway (a pathway linked to both inflammation and apoptosis) [32]; however, to our knowledge, there is no study directly examining the expression of myostatin in renal microcirculatory cells.

An experimental study exploited a genetic modified- FasL deficient murine model with renal cystic disease [33]. This study demonstrated that FasL-Fas interaction is not participating in the c-myc induced apoptosis in this model [33]. However, as the authors stated, these results could not rule out the participation of FasL-Fas in renal apoptotic mechanisms in renal cystic disease, as there are previous reports demonstrating alternative complex suicidal mechanisms driven by FasL-Fas [34]. Furthermore, sFasL has been linked to apoptotic mechanisms in other experimental setting or disease states. Of great interest, it has been indicated that sFasL could be linked to apoptosis of lung epithelial cells, in patients with acute respiratory distress syndrome [19]. In addition, previous data demonstrated that sFasL could potently induce cardiomyocyte apoptosis in patients with acute myocardial infarction (AMI) [20]. Plasma sFasL levels in AMI patients were also elevated, and authors suggested that increased sFasL expression could be related to ischemia/reperfusion injury in this patient group [20].

In our study, we demonstrated that beyond ADPKD patients with impaired renal function, patients at early stages of the disease had significantly higher levels of FasL 


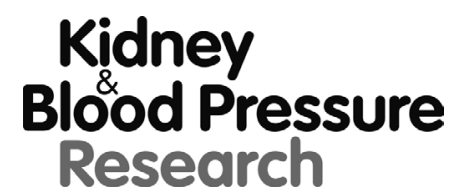

\begin{tabular}{l|l}
\hline Kidney Blood Press Res 2018;43:744-754 \\
\hline DOI: 10.1159/000489911 & (c) 2018 The Author(s). Published by S. Karger AG, Basel
\end{tabular}

and myostatin compared to controls. As it was expected, FasL and myostatin levels were negatively correlated with renal function, accessed via eGFR in patient group, but they were also positively correlated with ADMA, as happened in diabetic nephropathy subjects [17]. Therefore, this is the first observation in humans with ADKPD that endothelial dysfunction, via local microcirculatory changes, as above discussed, provokes mechanisms related to apoptosis even from the early stages of the disease.

Interstitial fibrosis is considered a key morphological change in ADPKD, which alongside with other local disturbances, such as inflammation, are contributing to decline of renal function [5]. As it is widely known, the TGF beta system is considered as key participant and regulator in fibrotic changes in renal parenchyma in various renal diseases, including primary glomerulopathies and diabetic nephropathy; urinary TGF- $\beta 1$ levels have been used as a marker reflecting fibrotic changes, and are proposed as diagnosis indicators of interstitial injury $[23-25,35,36]$. Again our study data showed that not only ADPKD patients with impaired renal function, as expected, but most importantly ADPKD patients with preserved renal function and equal eGFR and similar demographic and clinical characteristics with controls had significantly increased urinary TGF- $\beta 1$. Correlation analysis demonstrated that TGF- $\beta 1$ was negatively associated with eGFR and positively associated with ADMA in ADPKD patients. Strikingly, multivariate analysis demonstrated FasL as an independent predictor of increased urinary TGF- $\beta 1$ levels in ADPKD population. ADMA was also indicated by multivariate analysis as a potent factor related to increased urinary TGF- $\beta 1$ levels in ADPKD patients, although with a borderline statistical significance. These observations strongly support the aforementioned hypothesis on the close association of endothelial microcirculatory dysfunction with ischemia, apoptosis and renal fibrotic damage in these patients.

This is the first study examining emerging biomarkers related to apoptosis and fibrosis, such as FasL, myostatin and urine TGF- $\beta 1$, and their associations in patients at early stages of ADPKD. However, some limitations could be acknowledged. Our sample size could be considered small; however, all of our observations were highly significant. We went through a careful matching process to form ADPKD groups different only for eGFR, but there were inevitable other differences, such as prevalence of hypertension and albuminuria. We have managed, however, to have the group of ADPKD patients with preserved renal function wellbalanced with the control group in terms of all the main factors examined. It should be also mentioned, that this was a cross-sectional study, and thus, cause-and-effect associations cannot be established.

\section{Conclusion}

This study investigated serum FasL, serum myostatin and urine TGF- $\beta 1$, as biomarkers related to apoptosis and fibrosis, in patients with ADPKD of different stages and how these relate to endothelial dysfunction. We observed that, not only patients with low eGFR, but also those at early stages of the disease had higher expression of these biomarkers indicative in comparison to controls, and that both apoptotic and fibrotic markers were strongly associated with ADMA, an established marker of endothelial dysfunction. Taking into account previous observations from our group, these findings further support the hypothesis that, beyond cyst growth, local microcirculatory changes related to endothelial dysfunction cause hypoxia and renal ischemia, facilitating the activation of apoptosis and fibrosis in the renal tissue even from the early stages of ADPKD. Given the complexity of the disease, but also the need to further broaden our current knowledge, more experimental, mechanistic, but also large scale clinical studies are needed to generate new therapeutic strategies for ADKPD. 


\section{Kidney \\ Blood Pressure Research}

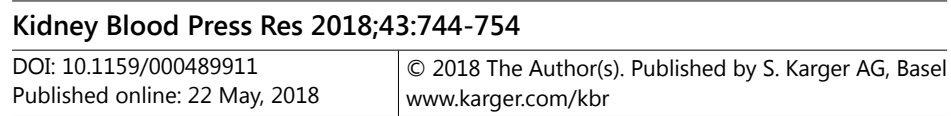

Raptis et al.: Apoptosis, Fibrosis and Endothelial Dysfunction in ADPKD

\section{Disclosure Statement}

The authors declare they have no conflicts of interest regarding this article.

\section{References}

1 Grantham JJ, Chapman AB, Torres VE: Volume progression in autosomal dominant polycystic kidney disease: the major factor determining clinical outcomes. Clin J Am Soc Nephrol 2006;1:148-157.

-2 Torres VE, King BF, Chapman AB, Brummer ME, Bae KT, Glockner JF, Arya K, Risk D, Felmlee JP, Grantham JJ, Guay-Woodford LM, Bennett WM, Klahr S, Meyers CM, Zhang X, Thompson PA, Miller JP: Magnetic resonance measurements of renal blood flow and disease progression in autosomal dominant polycystic kidney disease. Clin J Am Soc Nephrol 2007;2:112-120.

-3 Mao Z, Chong J, Ong AC: Autosomal dominant polycystic kidney disease: recent advances in clinical management. F1000Res 2016;5:2029.

-4 Torres VE, Chapman AB, Devuyst O, Gansevoort RT, Perrone RD, Koch G, Ouyang J, McQuade RD, Blais JD, Czerwiec FS, Sergeyeva 0: Tolvaptan in Later-Stage Autosomal Dominant Polycystic Kidney Disease. N Engl J Med 2017;377:1930-1942.

-5 Raptis V, Loutradis C, Sarafidis PA: Renal injury progression in autosomal dominant polycystic kidney disease: a look beyond the cysts. Nephrol Dial Transplant 2018; DOI: 10.1093/ndt/gfy023.

-6 Saran R, Robinson B, Abbott KC, Agodoa LY, Albertus P, Ayanian J, Balkrishnan R, Bragg-Gresham J, Cao J, Chen JL, Cope E, Dharmarajan S, Dietrich X, Eckard A, Eggers PW, Gaber C, Gillen D, Gipson D, Gu H, Hailpern SM, et al.: US Renal Data System 2016 Annual Data Report: Epidemiology of Kidney Disease in the United States. Am J Kidney Dis 2017;69:A7-A8.

7 Ong AC, Harris PC: A polycystin-centric view of cyst formation and disease: the polycystins revisited. Kidney Int 2015;88:699-710.

-8 Takakura A, Contrino L, Zhou X, Bonventre JV, Sun Y, Humphreys BD, Zhou J: Renal injury is a third hit promoting rapid development of adult polycystic kidney disease. Hum Mol Genet 2009;18:2523-2531.

-9 Happe H, Leonhard WN, van der Wal A, van de Water B, Lantinga-van Leeuwen IS, Breuning MH, de Heer E, Peters DJ: Toxic tubular injury in kidneys from Pkd1-deletion mice accelerates cystogenesis accompanied by dysregulated planar cell polarity and canonical Wnt signaling pathways. Hum Mol Genet 2009;18:25322542.

10 Antignac C, Calvet JP, Germino GG, Grantham JJ, Guay-Woodford LM, Harris PC, Hildebrandt F, Peters DJ, Somlo S, Torres VE, Walz G, Zhou J, Yu AS: The Future of Polycystic Kidney Disease Research--As Seen By the 12 Kaplan Awardees. J Am Soc Nephrol 2015;26:2081-2095.

-11 AbouAlaiwi WA, Takahashi M, Mell BR, Jones TJ, Ratnam S, Kolb RJ, Nauli SM: Ciliary polycystin-2 is a mechanosensitive calcium channel involved in nitric oxide signaling cascades. Circ Res 2009;104:860-869.

12 Nauli SM, Kawanabe Y, Kaminski JJ, Pearce WJ, Ingber DE, Zhou J: Endothelial cilia are fluid shear sensors that regulate calcium signaling and nitric oxide production through polycystin-1. Circulation 2008;117:1161-1171.

-13 Raptis V, Georgianos PI, Sarafidis PA, Sioulis A, Makedou K, Makedou A, Grekas DM, Kapoulas S: Elevated asymmetric dimethylarginine is associated with oxidant stress aggravation in patients with early stage autosomal dominant polycystic kidney disease. Kidney Blood Press Res 2013;38:72-82.

-14 Schneider P, Bodmer JL, Holler N, Mattmann C, Scuderi P, Terskikh A, Peitsch MC, Tschopp J: Characterization of Fas (Apo-1, CD95)-Fas ligand interaction. J Biol Chem 1997;272:18827-18833.

15 Micheau 0, Tschopp J: Induction of TNF receptor I-mediated apoptosis via two sequential signaling complexes. Cell 2003;114:181-190.

16 Dounousi E, Koliousi E, Papagianni A, Ioannou K, Zikou X, Katopodis K, Kelesidis A, Tsakiris D, Siamopoulos KC: Mononuclear leukocyte apoptosis and inflammatory markers in patients with chronic kidney disease. Am J Nephrol 2012;36:531-536. 


\section{Kidney \\ Blood Pressure Research}

Raptis et al.: Apoptosis, Fibrosis and Endothelial Dysfunction in ADPKD

17 Yilmaz MI, Sonmez A, Saglam M, Yaman H, Cayci T, Kilic S, Eyileten T, Caglar K, Oguz Y, Vural A, Yenicesu M, Axelsson J: Reduced proteinuria using ramipril in diabetic CKD stage 1 decreases circulating cell death receptor activators concurrently with ADMA. A novel pathophysiological pathway? Nephrol Dial Transplant 2010;25:3250-3256.

18 Brown SB, Savill J: Phagocytosis triggers macrophage release of Fas ligand and induces apoptosis of bystander leukocytes. J Immunol 1999;162:480-485.

-19 Matute-Bello G, Liles WC, Steinberg KP, Kiener PA, Mongovin S, Chi EY, Jonas M, Martin TR: Soluble Fas ligand induces epithelial cell apoptosis in humans with acute lung injury (ARDS). J Immunol 1999;163:2217-2225.

20 Shimizu M, Fukuo K, Nagata S, Suhara T, Okuro M, Fujii K, Higashino Y, Mogi M, Hatanaka Y, Ogihara T: Increased plasma levels of the soluble form of Fas ligand in patients with acute myocardial infarction and unstable angina pectoris. J Am Coll Cardiol 2002;39:585-590.

-21 Yang W, Zhang Y, Li Y, Wu Z, Zhu D: Myostatin induces cyclin D1 degradation to cause cell cycle arrest through a phosphatidylinositol 3-kinase/AKT/GSK-3 beta pathway and is antagonized by insulin-like growth factor 1. J Biol Chem 2007;282:3799-3808.

-22 Yano S, Nagai A, Isomura M, Yamasaki M, Kijima T, Takeda M, Hamano T, Nabika T: Relationship between Blood Myostatin Levels and Kidney Function:Shimane CoHRE Study. PLoS One 2015;10:e0141035.

23 Prakash J, de Borst MH, van Loenen-Weemaes AM, Lacombe M, Opdam F, van Goor H, Meijer DK, Moolenaar F, Poelstra K, Kok RJ: Cell-specific delivery of a transforming growth factor-beta type I receptor kinase inhibitor to proximal tubular cells for the treatment of renal fibrosis. Pharm Res 2008;25:2427-2439.

24 Chihara Y, Ono H, Ishimitsu T, Ono Y, Ishikawa K, Rakugi H, Ogihara T, Matsuoka H: Roles of TGF-beta1 and apoptosis in the progression of glomerulosclerosis in human IgA nephropathy. Clin Nephrol 2006;65:385392.

-25 Chen S, Jim B, Ziyadeh FN: Diabetic nephropathy and transforming growth factor-beta: transforming our view of glomerulosclerosis and fibrosis build-up. Semin Nephrol 2003;23:532-543.

-26 Ravine D, Gibson RN, Walker RG, Sheffield LJ, Kincaid-Smith P, Danks DM: Evaluation of ultrasonographic diagnostic criteria for autosomal dominant polycystic kidney disease 1. Lancet 1994;343:824-827.

27 Group. KCW: KDIGO 2012 clinical practice guidelines for the evaluation and management of chronic kidney disease. Kidney Int Suppl 2013;3:1-150.

28 Tsang TS, Barnes ME, Gersh BJ, Bailey KR, Seward JB: Left atrial volume as a morphophysiologic expression of left ventricular diastolic dysfunction and relation to cardiovascular risk burden. Am J Cardiol 2002;90:1284-1289.

29 Levey AS, Bosch JP, Lewis JB, Greene T, Rogers N, Roth D: A more accurate method to estimate glomerular filtration rate from serum creatinine: a new prediction equation. Modification of Diet in Renal Disease Study Group. Ann Intern Med 1999;130:461-470.

30 Raptis V, Loutradis C, Sarafidis PA: Renal injury progression in autosomal dominant polyscystic kidney disease: a look beyond the cysts. Nephrol Dial Transplant 2018; DOI: 10.1093/ndt/gfy023.

-31 Raptis V, Bakogiannis C, Loutradis C, Boutou AK, Lampropoulou I, Intzevidou E, Sioulis A, Balaskas E, Sarafidis PA: Levels of endocan, angiopoetin-2 and HIF-1a in patients with autosomal dominant polycystic kidney disease and different levels of renal function. Am J Nephrol 2018;47:231-238.

-32 Dhanasekaran DN, Reddy EP: JNK signaling in apoptosis. Oncogene 2008;27:6245-6251.

-33 Couillard M, Guillaume R, Tanji N, D’Agati V, Trudel M: c-myc-induced apoptosis in polycystic kidney disease is independent of FasL/Fas interaction. Cancer Res 2002;62:2210-2214.

34 Hueber AO, Zornig M, Lyon D, Suda T, Nagata S, Evan GI: Requirement for the CD95 receptor-ligand pathway in c-Myc-induced apoptosis. Science 1997;278:1305-1309.

35 Grenda R, Wuhl E, Litwin M, Janas R, Sladowska J, Arbeiter K, Berg U, Caldas-Afonso A, Fischbach M, Mehls O, Sallay P, Schaefer F: Urinary excretion of endothelin-1 (ET-1), transforming growth factor- beta1 (TGFbeta1) and vascular endothelial growth factor (VEGF165) in paediatric chronic kidney diseases: results of the ESCAPE trial. Nephrol Dial Transplant 2007;22:3487-3494.

-36 Tsapenko MV, Nwoko RE, Borland TM, Voskoboev NV, Pflueger A, Rule AD, Lieske JC: Measurement of urinary TGF-beta1 in patients with diabetes mellitus and normal controls. Clin Biochem 2013;46:14301435. 